\title{
ELASTIC PROPERTIES OF CROSSLINKED POLYMERS
}

\author{
G. REHAGE \\ Institute of Physical Chemistry, Technical University, Clausthal, \\ 3392 Clausthal-Zellerfeld, GFR
}

\begin{abstract}
At first a survey is given on the thermodynamics of rubber-elasticity and the results of the statistical theory of molecular networks. Experimental data of torsional vibrations, stress-strain measurements (simple extension and uniaxial compression) and photoelastic properties in dependence of temperature are reported. The measurements have been carried out on dry and swollen crosslinked polymers, especially poly(methyl methacrylate) (crosslinked in bulk or in solution), natural rubber, trans-polypentenamer and poly(butadiene). The modulus-temperature curves were always linear in the rubbery region when the samples had been carefully extracted. For poly(methyl methacrylate) the curve of retractive torce against temperature showed a bend at a distinct temperature. Below this temperature the slope of the curve is lower than above. This behaviour can be explained by the existence of long living associations in poly(methyl methacrylate)-solvent systems already known by measurements on the corresponding solutions. The associations are able to form physical crosslinks.

Most elastomers do not behave ideally in the sense of the theory: they are not purely entropy-elastic but show also an energy elasticity term of the modulus. The energy elasticity part is not constant but increases with the network density and the degree of swelling. From this it is concluded that the energy elasticity contribution is not only an intramolecular effect caused by rotational isomerism. The energy elasticity may also depend on other intramolecular effects and intermolecular sources. The existence of short chains in the network seems to be one essential reason for the nonconstancy of the energy elasticity contribution. Photoelastic measurements in dependence of the degree of swelling show that we must assume a short-range order in amorphous polymers. Following a newer theoretical consideration, the second term of the Mooney Rivlin equation for the stress-strain behaviour of unswollen elastomers can be explained by the co-operation of the short-range order and the chain-length distribution in the random polymer network.
\end{abstract}

\section{INTRODUCTION}

Here we will deal only with the elastic properties above the glass- or melting-temperature; that is, we will consider the hehaviour of polymers in the rubbery state. In the highly elastic state the substance consists of a three-dimensional macromolecular network. The crosslinks can be formed by primary bonds, secondary bonds, entanglements or domains in copolymers, which are due to phase-separation ${ }^{1,2}$. The primary bonds are chemical 


\section{G. REHAGE}

bonds, which cannot be detached without chemical reaction. The secondary bonds are physical bonds, which may be loosened or knotted by temperature or concentration changes. The physical bonds consist mainly of crystalline regions, eventually of stable associations. All these network structures can overlap each other. In the following we will consider mainly networks formed by chemical crosslinking.

\section{THEORETICAL CONSIDERATIONS ${ }^{3}$ 8}

\section{Thermodynamic treatment}

Now. I would like to refer to the theoretical aspects. At first we have to discuss the thermodynamic behaviour of a crosslinked system. Let us assume a homogeneous body. which is subjected to a simple extension or uniaxial compression. Then in the simplest case, the Gibbs equation will have on the assumption of volume constancy the following form:

$$
\mathrm{d} U=T \mathrm{~d} S+f \mathrm{~d} l ; \mathrm{d} V=0
$$

$U$ is the internal energy, $T$ the absolute temperature, $S$ the entropy, $f$ the force. $l$ the length and $V$ the volume of the system.

From equation (1) follows :

$$
\left(\begin{array}{l}
\hat{C} U \\
\partial i
\end{array}\right)_{V \cdot T}=T\left(\begin{array}{l}
\partial S \\
\partial l
\end{array}\right)_{v \cdot T}+f
$$

By means of pure thermodynamics one gets:

$$
\left(\begin{array}{c}
\partial S \\
\partial l
\end{array}\right)_{V . T}=-\left(\begin{array}{l}
\partial f \\
\partial T
\end{array}\right)_{V . l}
$$

Combinations of equations (2) and (3) gives:

$$
\left(\begin{array}{c}
\partial U \\
\partial l
\end{array}\right)_{V . T}=-T\left(\begin{array}{c}
\partial f \\
\partial T
\end{array}\right)_{V . l}+f
$$

$f \mathrm{~d} /$ has the sense of the reversible deformation work. $\mathrm{d} W_{\mathrm{rcv}}$, which is a fictitious work. This means that at any time the retractive force $f$ in the system is equal to the force applied to the system. which is actuatly not possible in the course of a real process. The formulas are always applicable if the state of the system can be completely described with two of the variables $f . l$ and $T$. The formulas cannot be applied if relaxation processes occur. In this case one needs further (internal) variables. But also then they can be used in the integrated form, if the beginning and the end of the process are completely defined by two of the variables $f, l$ and $T$. Between these variables exists a function of state like the ideal gas law in gaseous systems.

An ideal rubber is defined by:

$$
\left(\begin{array}{c}
\partial U \\
l l
\end{array}\right)_{v, T}=0
$$

Then it follows from equation (4) that

$$
f^{\prime}=\mathrm{a} \cdot T ; \text { where } \mathbf{a} \text { is a constant }
$$




\section{ELASTIC PROPERTIES OF CROSSLINKED POLYMERS}

In this case we have only entropy elasticity. But in most cases a rubber does not show only entropy elasticity but also energy elasticity, which seems to be independent of temperature. Then we find:

$$
f=f_{U}+f_{\mathrm{S}}=\mathrm{b}+\mathrm{a} T \text {; where } \mathrm{b} \text { is a constant }
$$

$f_{U}$ is the energy and $f_{S}$ the entropy contribution to the retractive force $f$.

In this elementary treatment volume constancy is assumed. Direct measurements at constant volume have been carried out by Allen et al. ${ }^{1 \mathrm{t}}$. It is, however, a difficult task to carry out measurements on solids at constant volume. It may be easier to perform the extension or compression measurements at constant pressure and to find the corresponding values at constant volume by calculation.

To obtain exact elastic parameters it seems better to perform shear or torsional measurements because shear and torsion are constant volume deformations on classical elasticity theory ${ }^{8}$. This is not quite correct, as Flory and Treloar have shown, but the volume changes are of second order and the difference between constant volume and pressure coefficients is simpler ${ }^{9.10}$. Therefore, torsion can provide a much more accurate basis for deriving the internal-energy contribution from experiments at constant pressure than does simple extension.

In the case of pure shear or torsion one obtains for the fictitious reversible work per volume unit (form of sample : circular cylinder) :

$$
\mathrm{d} W_{V, \text { rev }}=\mathrm{d}\left(\frac{W}{V}\right)_{\text {rev }}=G \cdot \gamma \cdot \mathrm{d} \gamma
$$

$G$ is the equilibrium shear or torsional modulus and $\gamma$ the shear strain.

The Gibbs equation now reads, for constant volume ${ }^{12}$ :

$$
\mathrm{d} U=T \mathrm{~d} S+V_{0} G \cdot \gamma \cdot \mathrm{d} \gamma ; \mathrm{d} V=0
$$

By pure thermodynamics one finds:

$$
\begin{aligned}
& \left(\frac{\partial F}{\partial \gamma}\right)_{V, T}=V_{0} \gamma \cdot G \\
& \left(\frac{\partial S}{\partial \gamma}\right)_{V, T}=-V_{0} \gamma\left(\frac{\partial G}{\partial T}\right)_{V \cdot \gamma} \\
& \left(\frac{\partial U}{\partial \gamma}\right)_{V \cdot T}=\left(\frac{\partial F}{\partial \gamma}\right)_{V \cdot T}+T\left(\frac{\partial S}{\partial \gamma}\right)_{V, T}=V_{0} \gamma G-V_{0} \gamma T\left(\frac{\partial G}{\partial T}\right)_{V, \gamma} \\
& \left(\frac{\partial U}{\partial \gamma}\right)_{V, T}=-V_{0} \gamma T^{2}\left[\frac{\partial}{\partial T}\left(\frac{G}{T}\right)\right]_{V \cdot \gamma}
\end{aligned}
$$

$F$ is the free energy. From equations(10)-(12) there follows:

$$
G=\frac{1}{V_{0} \gamma}\left[\left(\frac{\partial U}{\partial \gamma}\right)_{V, T}-T\left(\frac{\partial S}{\partial \gamma}\right)_{V, T}\right]=-T^{2}\left[\frac{\partial}{\partial T}\left(\frac{G}{T}\right)\right]_{V}+T\left(\frac{\partial G}{\partial T}\right)_{V}
$$

For linear-elastic behaviour, $G$ is independent of $\gamma$. Therefore we can omit the 


\section{G. REHAGE}

index $\gamma$ in equation (12). We call the first term in equation (13) the energy elastic term $G_{U}$ and the second term the entropy elastic term $G_{S}$ of the torsional (shear) modulus $G$.

$$
\begin{aligned}
& G=G_{U}+G_{S} \\
& G_{U}=\frac{1}{V_{0} \gamma}\left(\frac{\partial U}{\partial \gamma}\right)_{V \cdot T}=-T^{2}\left[\frac{\partial}{\partial T}\left(\frac{G}{T}\right)\right]_{V} \\
& G_{S}=-\frac{T}{V_{0} \cdot \gamma}\left(\frac{\partial S}{\partial \gamma}\right)_{V \cdot T}=T\left(\frac{\partial G}{\partial T}\right)_{V}
\end{aligned}
$$

With $G_{U}=0$ (ideal rubber) one obtains:

$$
G=G_{S}=\mathrm{a} T ; \text { where } \mathrm{a} \text { is a constant }
$$

With $G_{U}=$ b we have:

$$
G=\mathrm{b}+\mathrm{a} T ; \text { where } \mathrm{b} \text { is a constant }
$$

Of course the constants in this equation are not identical with those of equation (7).

If $G_{U}$ is temperature-dependent, $G$ becomes a complicated function of temperature.

\section{Results of the statistical theory}

The statistical thermodynamics gives an insight into the molecular base of the rubber elasticity. The classical theory of rubber elasticity yields for the equilibrium shear modulus at small deformations the well known formula ${ }^{8.9}$ :

$$
G=v k T \frac{\left\langle r^{2}\right\rangle}{\left\langle r_{0}{ }^{2}\right\rangle}
$$

$v$ is the number of elastically effective network chains per unit volume, $\boldsymbol{k}$ the Boltzmann factor, $\left\langle r^{2}\right\rangle$ the mean square end-to-end distance of network chains in the undeformed, unswollen state and $\left\langle r_{0}{ }^{2}\right\rangle$ the mean square end-to-end distance, for chains of the same length not constrained by crosslinks.

We have avoided all complications ${ }^{1}$ and the formula is therefore very simple. But the meaning of the factor $\left(v \cdot\left\langle r^{2}\right\rangle /\left\langle r_{0}{ }^{2}\right\rangle\right)$ is elucidated: at first one cannot separate the two terms of the parenthetical expression. Furthermore, Duiser and Staverman among others deduced ${ }^{13}$ that $v$ should be replaced by $v / 2$. Entanglements and other physical crosslinks are unknown quantities. The factor $\left\langle r^{2}\right\rangle\left\langle\left\langle r_{0}{ }^{2}\right\rangle\right.$ may be nearly 1 or smaller ${ }^{14}$. A comparison of the chemically and physically determined network density does not solve the problem. There may be more or fewer elastically effective crosslinks than found by chemical analysis.

For an elastomer swollen to the swelling degree $q=V / V_{0}\left(V_{0}=\right.$ volume in the dry state), one can derive for the shear modulus, the formula

$$
G_{q}=G \cdot q^{-1 / 3}
$$

if $\left\langle r_{0}{ }^{2}\right\rangle$ does not change during the swelling process, which can be assumed to be approximately true. In equation (17), $G$ refers to the dry state. 
For simple extension $(\lambda>1)$ or uniaxial compression $(\lambda<1)$ we get from theory:

$$
\begin{aligned}
& \sigma=v \boldsymbol{k} T \frac{\left\langle r^{2}\right\rangle}{\left\langle r_{0}{ }^{2}\right\rangle}\left(\lambda^{2}-\lambda^{-1}\right) \\
& \lambda=l / l_{0}
\end{aligned}
$$

$l$ is the length of the strained sample and $l_{0}$ the original length. The stress $\sigma$ is the force per unit cross-sectional area measured in the strained state $\lambda$ is the extension ratio. The factor $\left\langle r^{2}\right\rangle /\left\langle r_{0}{ }^{2}\right\rangle$ considers the case that the several rotational isomers of the polymer do not have the same internal energy. From this it follows that $\left\langle r_{0}{ }^{2}\right\rangle$ is temperature dependent and that in the strained state a part of the retractive force consists of a change of the internal energy with deformation.

If $\sigma_{0}$ is the force per unit area of the unstrained cross-section we have:

$$
\sigma_{0}=v k T \cdot \frac{\left\langle r^{2}\right\rangle}{\left\langle r_{0}{ }^{2}\right\rangle} \cdot\left(\lambda-\lambda^{-2}\right)
$$

For swollen elastomers we derive formulas, according equation (17), which contain the swelling degree or volume fraction of the polymer respectively.

A deformation is generally connected with a birefringence. In analogy with the derivation of the statistical theory of rubber elasticity, Kuhn and Grün derived a relation between the birefringence and the strain of an elastomer ${ }^{15}$. For this derivation the Lorentz and Lorenz equation is assumed valid.

The Kuhn and Grün equation reads, with regard to the dilatation factor ${ }^{16}$ :

$$
\Delta n=n_{1}-n_{2}=\frac{2 \pi}{45} \frac{\left(\bar{n}^{2}+2\right)^{2}}{\bar{n}} v \frac{\left\langle r^{2}\right\rangle}{\left\langle r_{0}{ }^{2}\right\rangle} . \Delta \alpha \cdot\left(\lambda^{2}-\lambda^{-1}\right)
$$

$\Delta n$ is the birefringence, that is the difference of the refractive indices in the direction of extension and perpendicular to it. $\bar{n}=\left(n_{1}+2 n_{2}\right) / 3$ is the mean refractive index and $\Delta \alpha$ the difference of the polarizabilities of the statistical segment in the direction of the segment and perpendicular to it. According to the statistical theory $\Delta n$ at constant strain should be nearly independent of the temperature.

The ratio of birefringence to stress is called the stress-optical coefficient $C$. From equations (18) and (20) there follows

$$
C=\frac{\Delta n}{\sigma}=\frac{2 \pi}{45 k T} \cdot \frac{\left(\bar{n}^{2}+2\right)^{2}}{\bar{n}} \cdot \Delta \alpha
$$

The formula shows that $C$ at a given temperature does not depend on the extension $\lambda$, the network density $v$ and the factor $\left\langle r^{2}\right\rangle\left\langle\left\langle r_{0}^{2}\right\rangle\right.$. For swollen samples this ratio is independent of the degree of swelling (except for a correction due to change in mean refractive index).

In these statistical theories only the properties of single polymer chains are taken into account. The interaction between the chains and volume requirements are not considered. Furthermore a Gaussian network is assumed: this means that the deformations should not be too large. 


\section{Viscoelastic properties}

Until now we have only considered the elastic behaviour of a crosslinked polymer. In the ideal case the elasticity consists only of entropy elasticity. Normaliy. as mentioned above, a rubbery material has additionally an energy elasticity contribution. Generally a polymer possesses elastic and viscous properties above the glass or melting temperature. In this connection we speak of a viscoelastic body. The properties of such a body are best and most easily determined by the dynamic-mechanical method. Mostly used are torsional vibration measurements. The viscoelastic body can be described by the complex shear modulus

$$
G^{*}=G^{\prime}+i G^{\prime \prime}(\text { where } i=\sqrt{ }-1)
$$

The storage modulus $G^{\prime}$ is a measure for the maximal stored reversible work of deformation per volume unit during a vibration period at the shear amplitude $\gamma=1$. The loss modulus $G^{\prime \prime}$ is a measure for the dissipated work per volume unit during a vibration period at the shear amplitude $\gamma=1^{12}$.

With $G^{\prime \prime}<G^{\prime}$ and the assumption that at low frequencies $G^{\prime}$ is independent of the frequency we can identify the storage modulus $G^{\prime}$ with the static shear modulus $G$. In this case we can perform a thermodynamic analysis using equations (8)-(15).

\section{SOME EXPERIMENTAL RESULTS AND DISCUSSION}

First we will discuss some newer results of torsional vibration measurements on rubbery substances. Some years ago a survey was made by Ferry ${ }^{17}$. Most measurements refer to dry elastomers. Only a few investigations have been made on swollen gels ${ }^{18.19}$.

In Figure 1 the storage modulus $G^{\prime}$ is plotted against temperature for various poly(methyl methacrylate) samples crosslinked in bulk ${ }^{12}$. Before starting the measurements the samples were carefully annealed but not extracted. In no case does the storage modulus increase with rising temperature, as predicted by the theory of rubber elasticity. The loss moduli $G^{\prime \prime}$ are of the

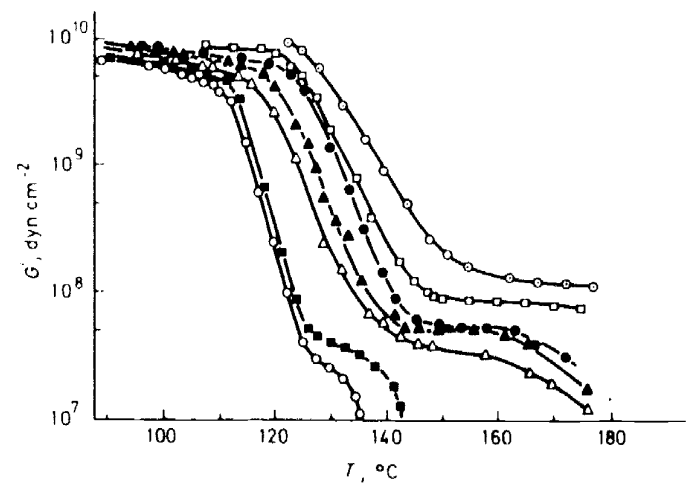

Figure 1. Plots against temperature of storage modulus $G^{\prime}$ for poly(methyl methaorylate) (PMMA) crosslinked with different amounts of ethylene glycol dimethacrylate (EGDM). Frequency $1 \mathrm{~Hz}{ }^{\prime}: 0.0: 0.06: \triangle 0.2 ; \Delta 1.0 ; 0 ; 0 ; \mid 8.0 ; \cdot 25$ weight \% EGDM. 
same magnitude as the $G^{\prime}$ moduli. The material is viscoelastic at a frequency of $1 \mathrm{~Hz}$.

Figure 2 shows the behaviour of one of the poly(methyl methacrylate) samples after being extracted with solvent and dried in vacuum. After this procedure we obtained quite different results. Above $170^{\circ} \mathrm{C}$ the storage modulus $G^{\prime}$ increases linearly with rising temperature. The loss modulus $G^{\prime \prime}$, however, decreased for some five decades compared with the same sample before extraction. Furthermore we plotted the values of $G^{\prime} / T$ against the temperature. If this curve is parallel to the temperature axis. the sample is ideally rubber elastic.

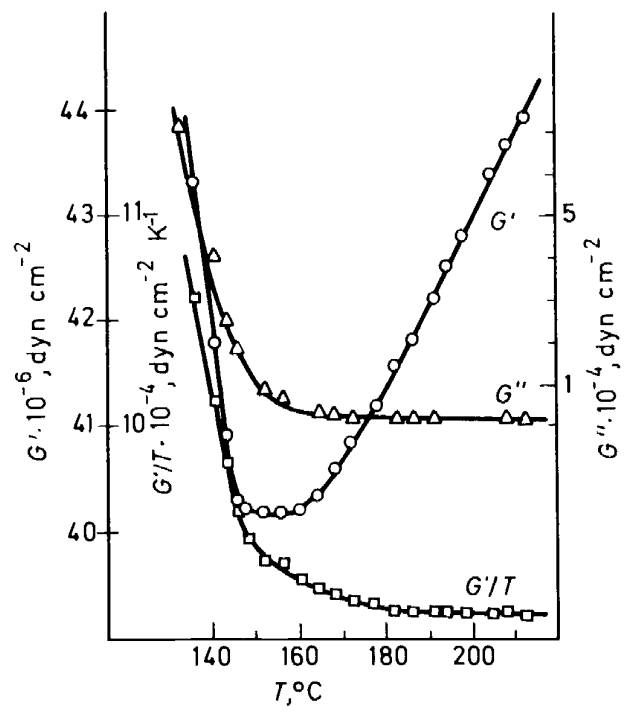

Figure 2. Plots against temperature of $G^{\prime}, G^{\prime \prime}$ and $G^{\prime} / T$ for a crosslinked PMMA sample, after extraction in $m$-xylene and drying. Frequency $1 \mathrm{~Hz} .8$ weight $\%$ of EGDM.

In Figure 3 is shown an example of torsional vibration measurements on swollen poly(methyl methacrylate). During the measurements the degree of swelling was kept constant. Now the glass temperature is 100 degrees lower than before.

For other poly(methyl methacrylate) samples crosslinked in bulk and crosslinked in chlorobenzene and then dried we obtained quite similar results (cf. Figure 4).

In the past, there have been many measurements of simple elongation, uniaxial compression, and torsion on natural rubber and other teehnically relevant elastomers by K. H. Meyer, Treloar, Flory, Gee, Allen, Krigbaum. Smith, Caspary $\mathrm{y}^{20}$ and others ${ }^{3,5,8}$. We have made torsional vibration measurements on severai elastomers of technical importance in the dry and swollen state ${ }^{12}$.

Without extraction we did not obtain equilibrium values and $G^{\prime}$ was not a linear function of the temperature. After extraction very accurate linear dependencies were obtained. 
G. REHAGE

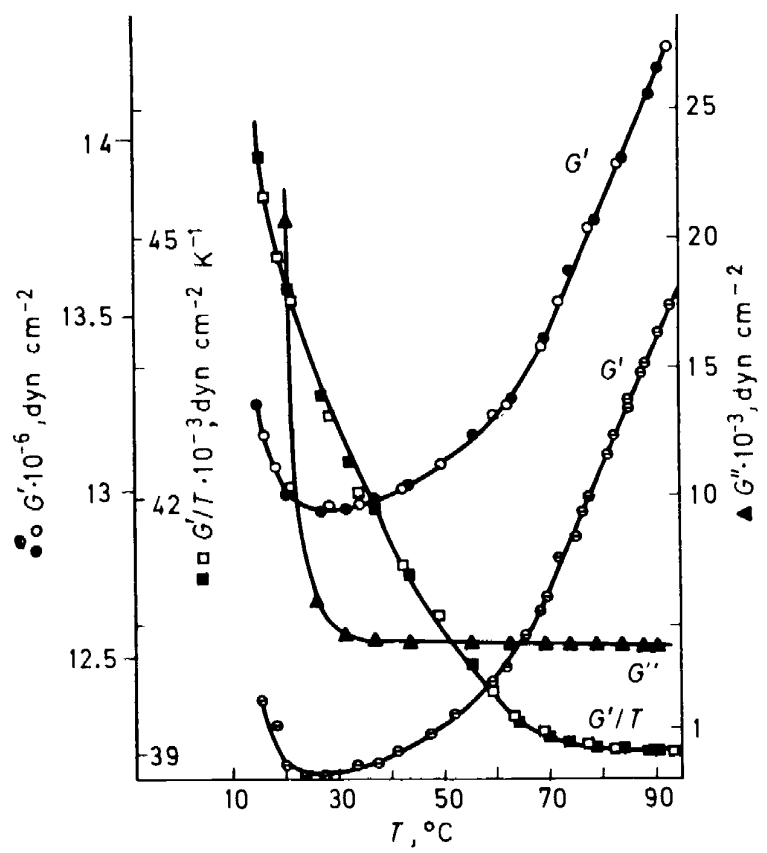

Figure 3. Plots against temperature of $G^{\prime}, G^{\prime \prime}$ and $G^{\prime} / T$ of a crosslinked PMMA sample, swollen in $m$-xylene. Frequency $0.5 \mathrm{~Hz} .1 \%$ EGDM.

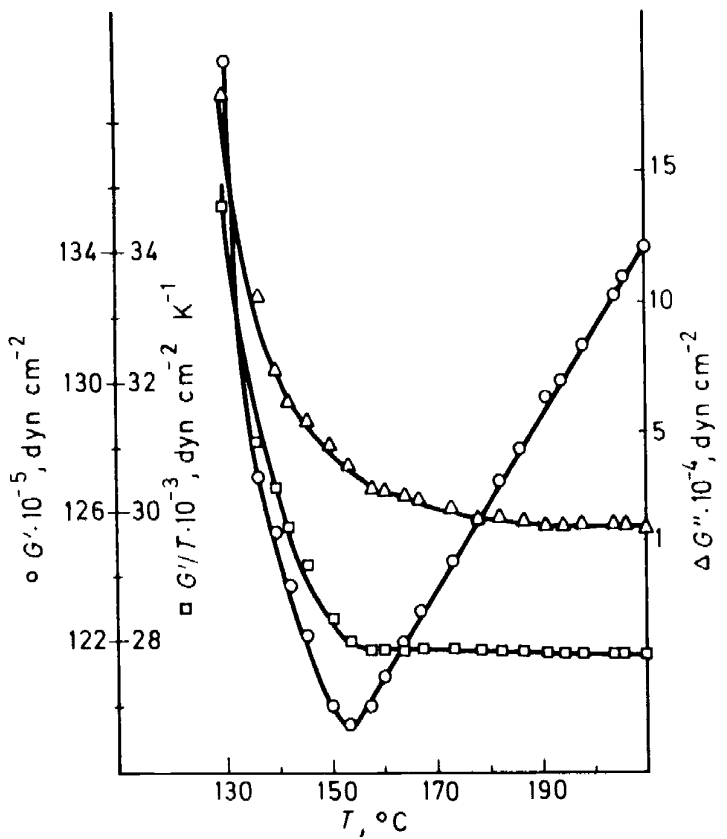

Figure 4. Plots against temperature of $G^{\prime}, G^{\prime \prime}$ and $G^{\prime} / T$ for a PMMA sample, crosslinked in chlorobenzene and dried. Frequency $1.0 \mathrm{~Hz} .5 \% \mathrm{EGDM}$ 
An example is given in Figure 5 : natural rubber, crosslinked with dicumyl peroxide, extracted into $m$-xylene and measured in the dry state. It is remarkable that a plot of $G^{\prime} / T$ against $T$ is not parallel to the temperature axis. This means that in this system the energy elasticity part $G_{U}^{\prime}$ of the storage modulus $G^{\prime}$ is very high.

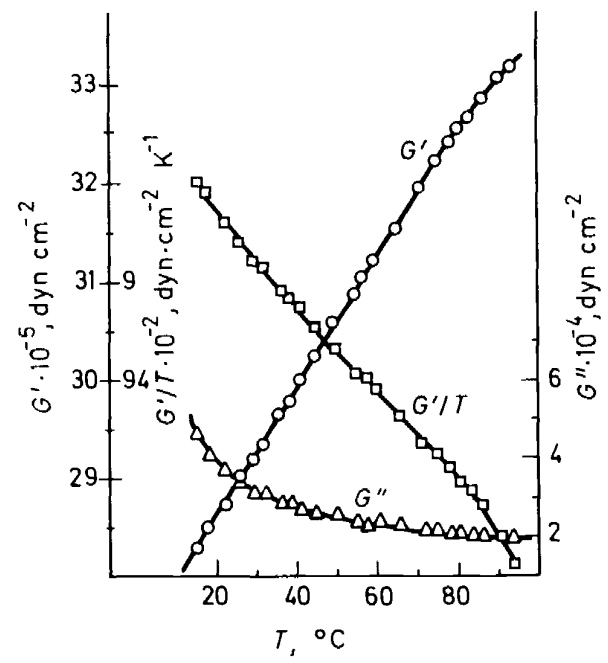

Figure 5. Plots against temperature of $G^{\prime}, G^{\prime \prime}$ and $G^{\prime} / T$ for a sample of natural rubber extracted and dried and crosslinked with $2 \mathrm{~g}$ of dicumyl peroxide (DCP) per $100 \mathrm{~g}$ of rubber. Frequency $0.9 \mathrm{~Hz}$

In Figure 6 are shown some torsional vibration measurements on trans1,5-polypentenamer (TPR) at several densities of crosslinking above the melting point. We achieved similar results with crosslinked poly(dimethylsiloxanes) ${ }^{21}$. All investigated elastomers, above the melting point and after

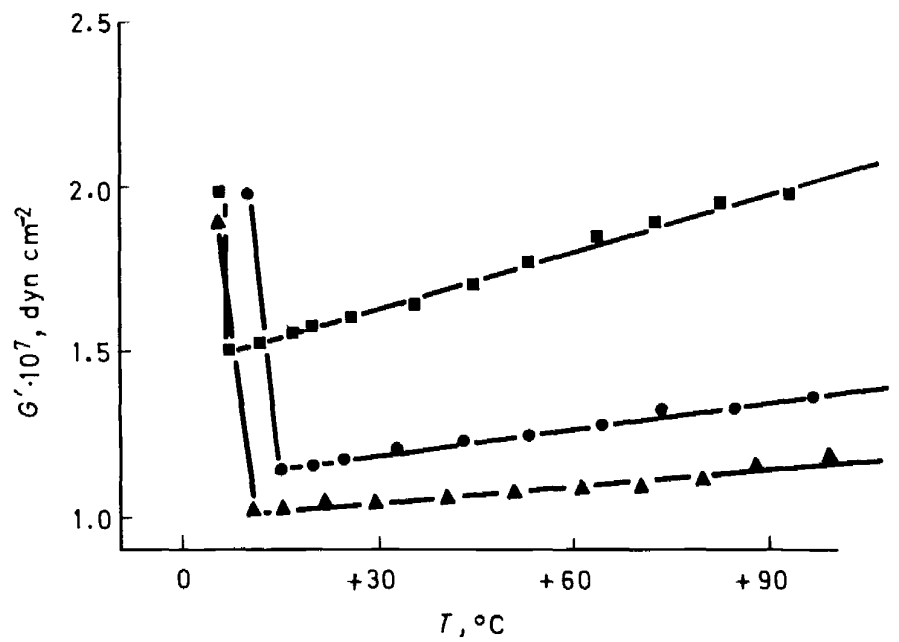

Figure 6. Plots against temperature of $G^{\prime}$ for trans-1,5-polypentenamer (TPR) crosslinked with DCP. $(\square 3 \% ; 1 \%: \triangle 0.5 \%$ DCP $)$ 


\section{G. REHAGE}

thorough and careful extraction, showed a linear dependence of the storage modulus $G^{\prime}$ on temperature.

By means of the statistical theories we can calculate the effective degree of crosslinking. But at first we have to test whether the dynamic shear modulus $G^{\prime}$ can be compared with the static equilibrium modulus $G$. Therefore, we determined the frequency dependence of $G^{\prime}$ and $G^{\prime \prime}$ for various elastomers $(0.1-1.8 \mathrm{~Hz})$. We found that $G^{\prime}$ and $G^{\prime \prime}$ were independent of the frequency in the range we used, providing the storage modulus rises linearly with temperature. Then. $G^{\prime}$ can be compared with the static modulus $G$ and we can calculate the crosslink density. For a given sample the result was independent of the sweliing agent within the errors of our experiments. So we can conclude that $\left\langle r_{0}{ }^{2}\right\rangle$ does not change strongly with the degree of swelling. But we cannot separate the factor $\left\langle r^{2}\right\rangle /\left\langle r_{0}{ }^{2}\right\rangle$ from the network density $v$. With $G^{\prime \prime}<G^{\prime}$ and frequency independence of $G^{\prime}$ it is possible to make the thermodynamic analysis and to determine the energy elasticity part of $G^{\prime}$. In Figure 7 the relative energy

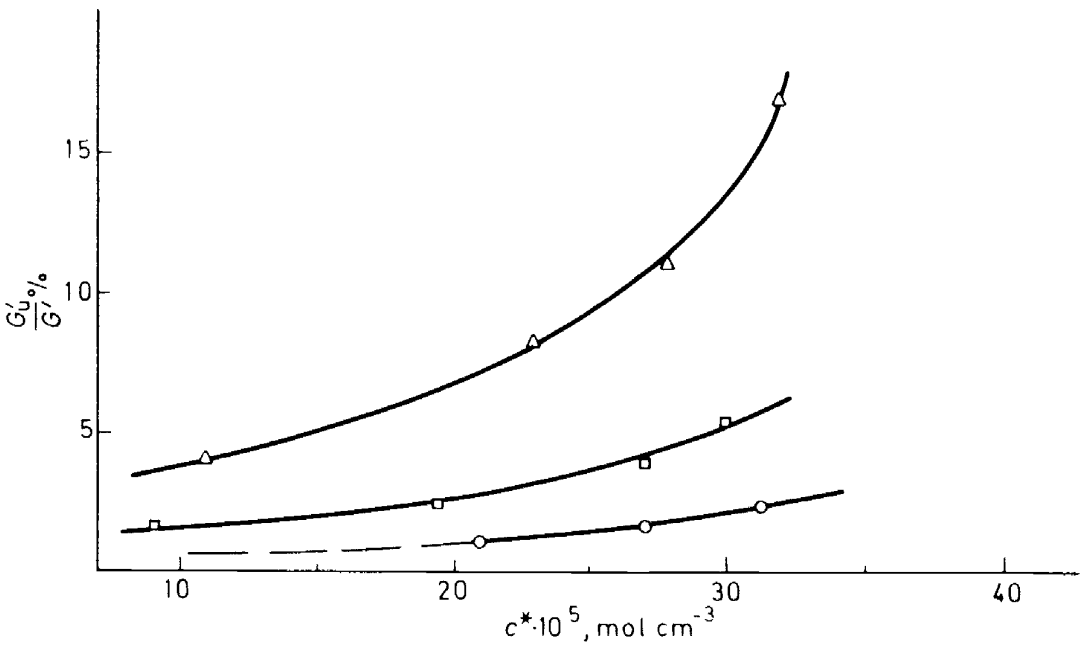

Figure 7. Relative energetic contribution of the storage modulus $\left(G_{U}^{\prime} / G^{\prime}\right.$ plotted against the effective network density $c^{*}=v N_{l}\left(N_{L}=\right.$ Avogadro number) for samples of $\mathrm{PMM} \Lambda$ polymerized in solution and crosslinked with EGDM. dry: swollen in $m$-xylene $; \wedge$ swollen in chlorobenzene

elasticity value $G_{U}^{\prime} / G^{\prime}$ is plotted against the effective degree of crosslinking for some dry and swollen poly(methyl methacrylate) samples. The energy elasticity component increases with rising density of crosslinking. For low density of crosslinking the samples showed almost ideal rubber elasticity.

In the next plot (Figure 8 ) is shown the energy elasticity term of the storage modulus as a function of the degree of swelling for poly(methyl methacrylate) samples. It was found that the energy elasticity component increases with rising degree of swelling.

The results for the natural rubber samples were similar.

We believe that the increase of the energy elasticity part $G_{U}^{\prime} / G^{\prime}$ with rising network density and swelling degree is caused by the short chains in the network. Usually a chain-length distribution exists in a network. Under strain, the chains, mostly the short ones, are extended. This explanation seems to be 


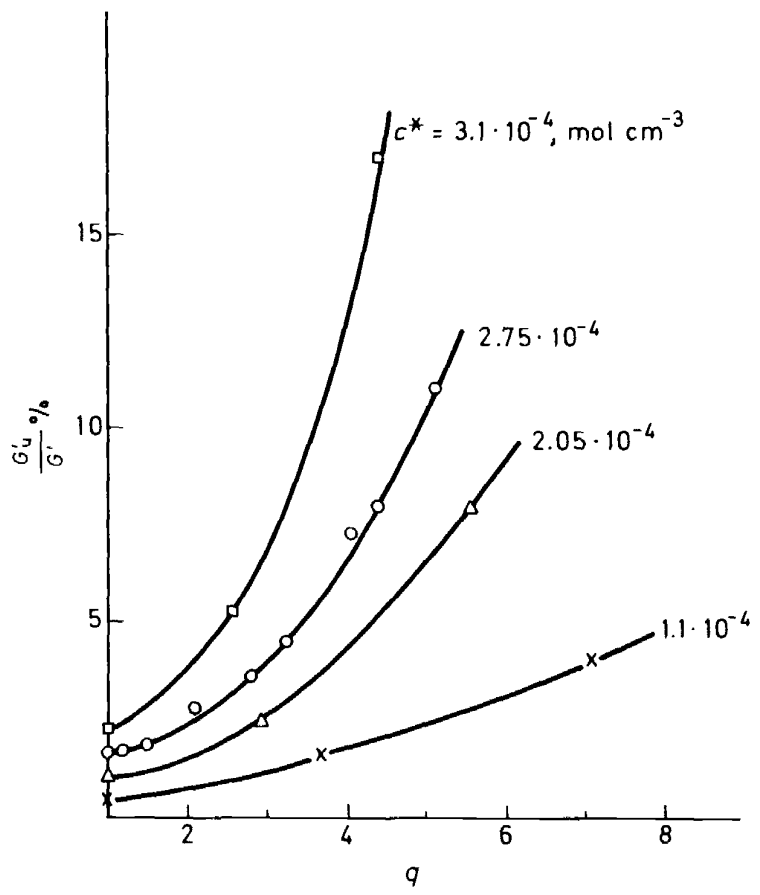

Figure 8. Relative energetic contribution of the storage modulus $G_{U}^{\prime} / G^{\prime}$ plotted against the degree of swelling $q=V / V_{0}$ for PMMA samples with different effective network density $c^{*}$ crosslinked in solution

supported by torsional vibration measurements on polybutadiene which is crosslinked by carboxylic end groups. These samples are, to a high degree, free from entanglements and short chains. The chain-length distribution is narrow. In these samples the relative energy elasticity part is nearly independent of the degree of swelling. They show ideal entropy elasticity at least for small deformations (Figure 9). Kraus and Moczygemba obtained similar results by means of stress-strain measurements ${ }^{22}$.

The origin of the energy elasticity is not completely clear. It may be positive (natural rubber, poly(methyl methacrylate) ${ }^{8,12}$ ) or negative ipolyethylene ${ }^{23}$, ethylene-(vinyl acetate) copolymer ${ }^{28}$ ). Most authors believe that only the temperature dependence of the mean square length $\left\langle r_{0}{ }^{2}\right\rangle$ of free chains is responsible for the energy elasticity according to the relation

$$
\frac{G_{U}}{G}=T \frac{\mathrm{d} \ln \left\langle r_{0}{ }^{2}\right\rangle}{\mathrm{d} T}
$$

which follows from equations (14b) and (16). Equation (23) has to do with the existence of rotational isomers and is therefore caused by intramolecular interaction ${ }^{24-26}$. From equation (23) it follows that $G_{U} / G$ should be constant. This is not always the case as is shown by our results on poly(methyl methacrylate) and natural rubber and force-temperature measurements performed by Opschoor and Prins on crosslinked polyethylene and ethylene- 


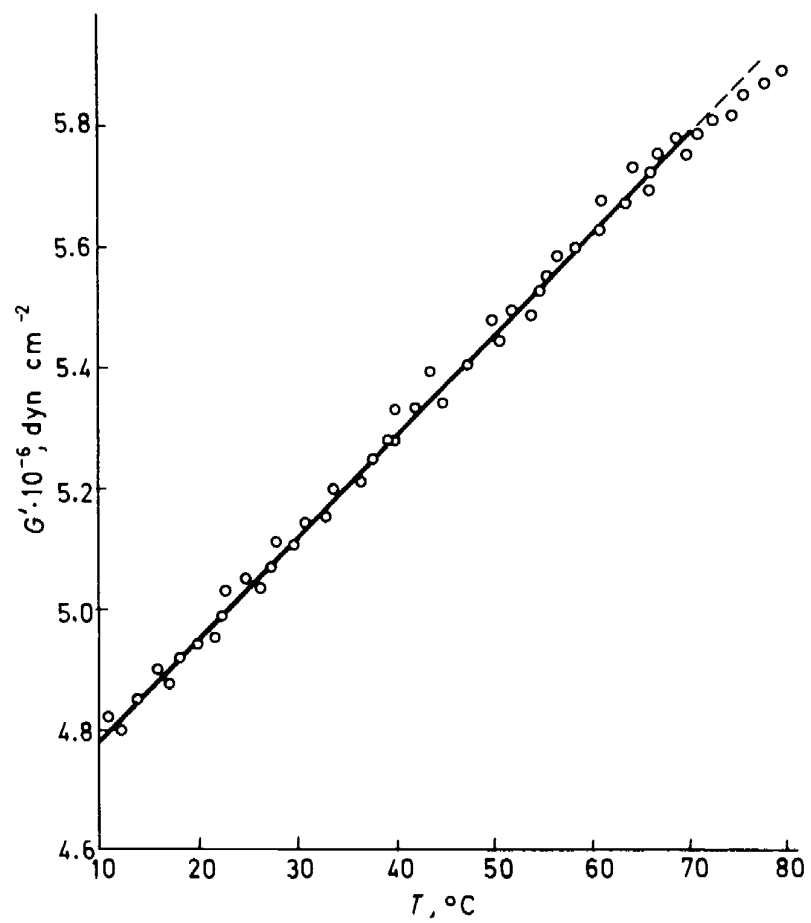

Figure 9. Temperature dependence of the storage modu'us $G^{\prime}$ of polybutadiene crosslinked over carboxylic end-groups $(3 \%$ of hexa[1-(2-methyl)aziridinyl]triphosphatriazine)

propylene copolymers ${ }^{23}$. Other authors confirm that the energy contribution to the retractive force depends on the extension ratio and the network density, respectively ${ }^{3,27,35}$. This consideration leads us to the assumption that the rotational isomerism may not be the only reason for the deviations of the ideal rubber behaviour. Besides intramolecular connections we must also take into account interactions between the molecules. Up to now intermolecular forces are not included in network theory. For an interpretation of the experimental results it may still be emphasized that it is difficult to estimate the role of faults in calculated or accepted volume constancy in these cases.

Now we will discuss the birefringence behaviour of uniaxial strained elastomers. Schwarz has made measurements on cis-1,4-polybutadiene and on a copolymer of butadiene and styrene ${ }^{29}$. Further measurements have been made on $\mathrm{TPR}^{30}$. All substances were crosslinked with dicumyl peroxide. We have seen that $\Delta n$ should be independent of temperature (equation 20). Figure 10 shows that this is not the case. $d_{0}$ is the width of the sample before the deformation and is introduced to simplify evaluation. We see that the birefringence at constant deformation decreases with increasing temperature. In Figure 11 the corrected stress optical coefficient $C^{\star}$ of swollen butadienestyrene copolymer is plotted against the swelling degree $q=\varphi_{2}^{-1}$. The correction refers to the mean refractive index $\bar{n}$ (cf. equation 21 ), which changes slightly with swelling. An isotropic solvent (carbon tetrachloride) was 


\section{ELASTIC PROPERTIES OF CROSSLINKED POLYMERS}

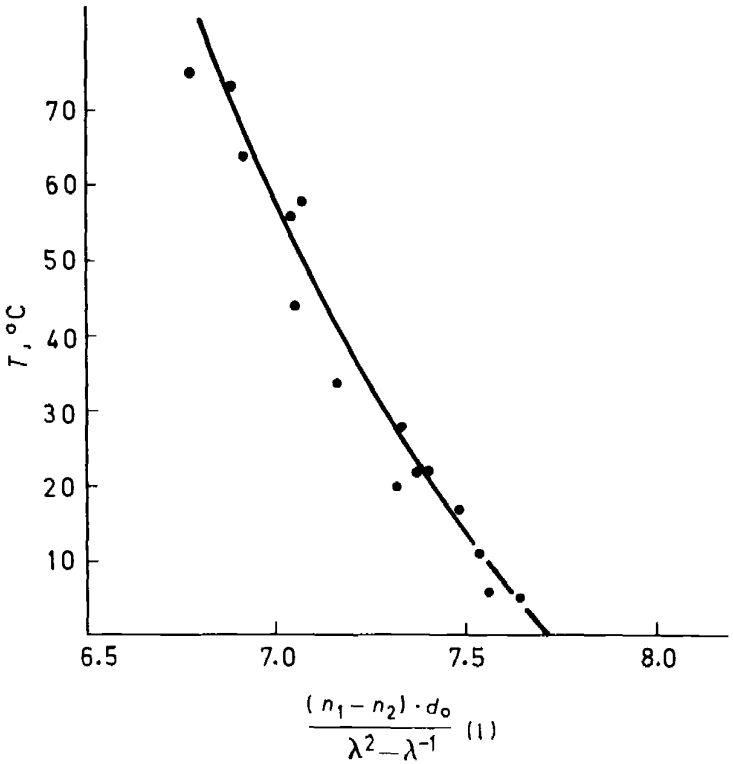

Figure 10. Temperature dependence of the expression $\left(n_{1}-n_{2}\right) d_{0} /\left(\lambda^{2}-\lambda^{-1}\right)$ for polybutadiene. $\lambda$ is the relative deformation, $\left(n_{1}-n_{2}\right)$ the birefringence and $d_{0}$ the width of the sample before the deformation

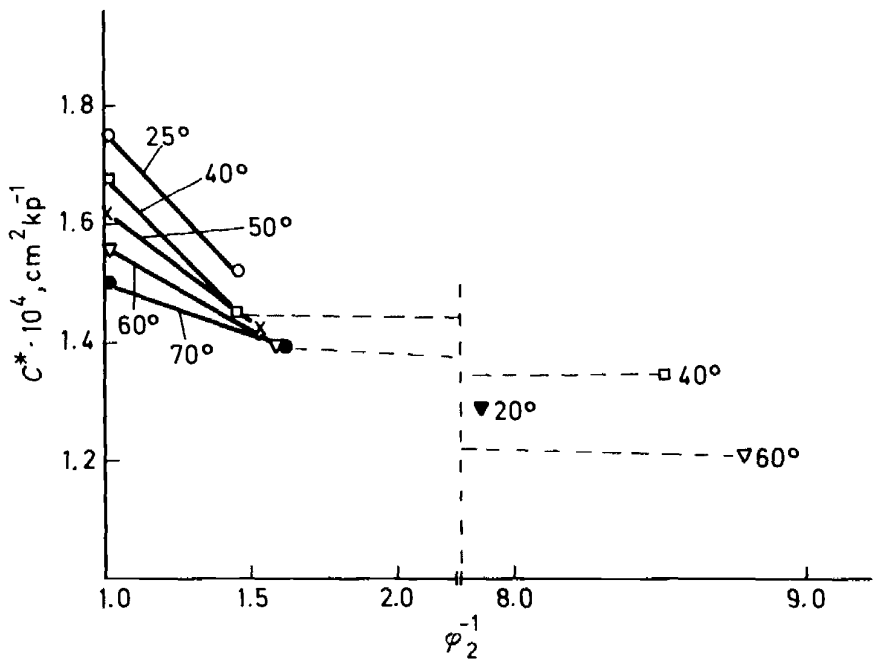

Figure 11. Corrected stress optical coefficient $C^{\star}$ as a function of the swelling degree $q=\varphi_{2}^{-1}$ of a butadiene-styrene copolymer ( $26 \%$ of polystyrene) at different temperatures. $\varphi_{2}$ is the volume fraction of polymer. Swelling agent, carbon tetrachloride 


\section{G. REHAGE}

chosen, to exclude secondary effects. Similar results have been obtained with all investigated elastomers and leads to the conclusion ${ }^{29.31}$ that a shortrange orientational order operates in amorphous polymers in such a way that adjacent segments of different chains prefer to be parallel to each other to meet volume or interaction requirements. This short-range order must decrease with increasing temperature and uptake of solvent. The influence of swelling shows that it is an intermolecular effect.

Let us consider another effect: The stress-strain behaviour of swollen crosslinked poly(methyl methacrylate) as function of the temperature is unusual. as seen in Figure $12^{32}$. Above $30^{\circ} \mathrm{C}$ the behaviour is normal. Below $30^{\circ} \mathrm{C}$ the modulus is greater and the reduced force-temperature curve becomes horizontal. We can explain this in the following way. We know that linear poly(methyl methacrylate) shows association, also in the atactic form ${ }^{33.34}$. Therefore we can assume that intermolecular associations are also present in crosslinked poly(methyl methacrylate). At lower temperatures these associations may be relatively stable in a network. So we will have a superposition of chemical and physical crosslinks. Therefore the plot of reduced force against

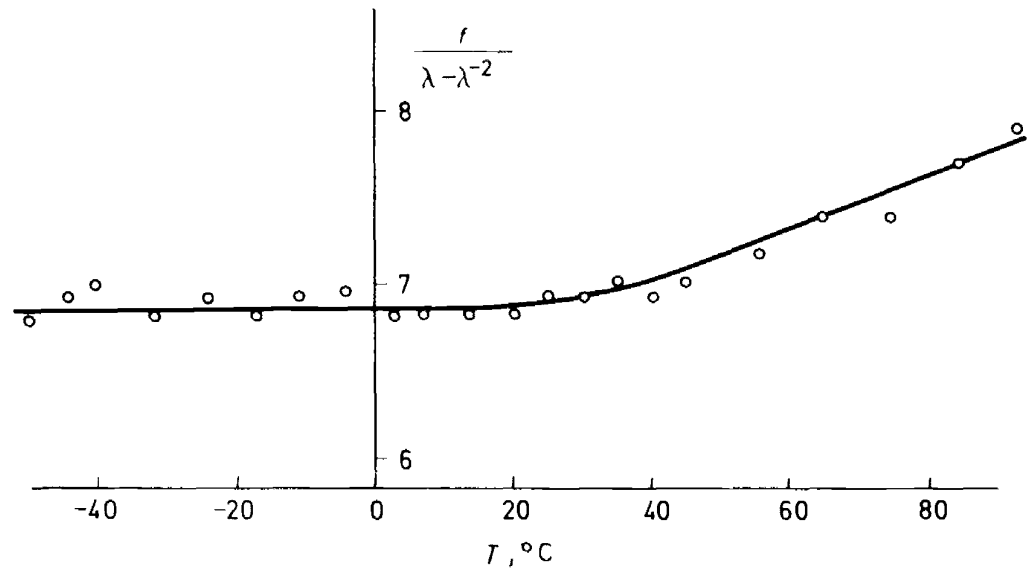

Figure 12. Reduced force $f / \lambda-\lambda^{2}$ as a function of temperature for a gel of crosslinked PMMA and chlorobenzene. Degree of swelling $q \approx 5$. Mean deformation nearly $7{ }^{\prime \prime}$.

temperature has a bend. We found this bend for all investigated poly(methyl methacrylate) samples. In contrary to this, gels from crosslinked polystyrene show normal temperature-dependence of the reduced force (Figure 13). From this we may conclude that for poly(methyl methacrylate) gels we can not determine the network density in a network of primary (chemical) crosslinks from the slope of the force-temperature plot at lower temperatures. To do so, we would have to take into account crosslinks of secondary (physical) bonds. It is necessary to measure the stress as a function of the temperature over such a large temperature range that we can exclude the existence of a bend and get only one straight line according to the theory. This holds for all polymers.

Mistures of isotactic and syndiotactic poly(methyl methacrylate) lead to formations of stereocomplexes in solution ${ }^{33.34}$. We suppose that this complex formation occurs in small regions also in so-called atactic poly(methyl 


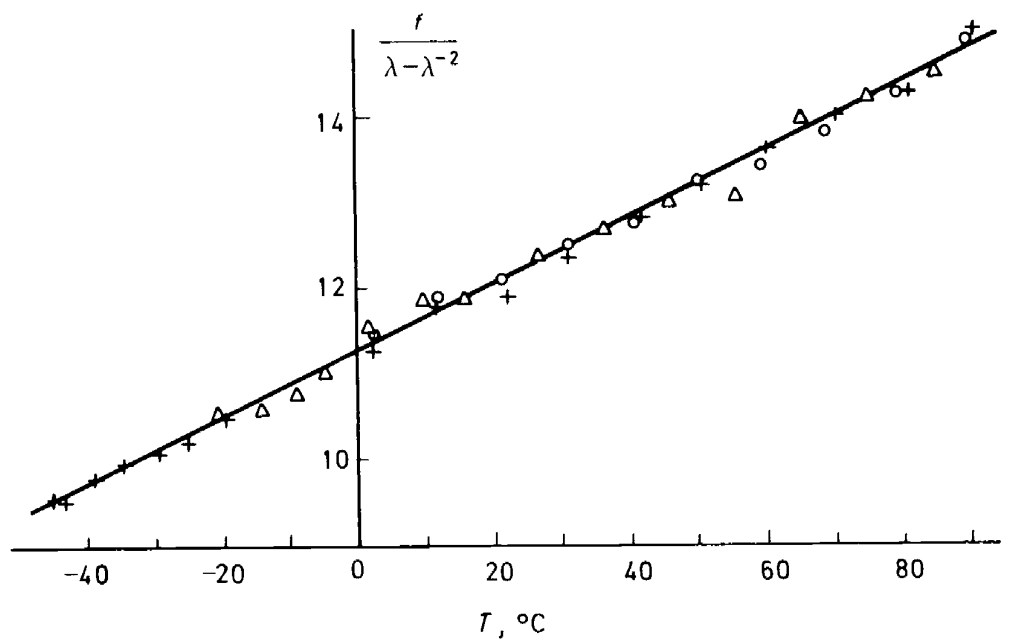

Figure 13. Reduced force $f / \lambda-\lambda^{-2}$ as a function of temperature for a gel of crosslinked polystyrene and chlorobenzene for several series of measurements with decreasing and increasing temperature. Degree of swelling $q \approx 10$. Mean deformation nearly $8 \%$

methacrylate) between tactic sequences. These complexes seem to form the long living intermolecular associations.

In the temperature region in which association leads to an elevated modulus, we cannot carry out thermodynamic analysis because we cannot describe the system only in terms of the two variables $T$ and $\lambda$.

It cannot be assumed that association equilibria are established. Therefore we have no true equilibrium and we must introduce further parameters, which describe the state of association.

\section{THE MOONEY-RIVLIN EQUATION}

Mooney and Rivlin have derived a phenomenological equation, which describes the stress-strain behaviour in a large deformation range (Figure 14).

$$
\sigma=C_{1}\left(\lambda^{2}-\lambda^{1}\right)+C_{2}\left(\lambda-\lambda^{-2}\right)
$$

with $C_{1}=v \boldsymbol{k} T\left\langle r^{2}\right\rangle /\left\langle r_{0}^{2}\right\rangle . C_{1}$ and $C_{2}$ are constants. The first term corresponds to the statistical theory. The second term gives a contribution to the retractive force, which becomes smaller or vanishes with increasing strain or swelling degree. Therefore the simplest behaviour according to the statistical theory is best fulfilled for swollen crosslinked polymers.

Many attempts have been made to explain the $C_{2}$ term on a molecular basis but without general acceptance ${ }^{1}$. The newest explanation has been given by Schwarz ${ }^{38}$. According to this theory the deviation from the statistical theory of rubber elasticity is explained by the cooperation of short-range order and chain-length distribution according to the following mechanism.

During deformation of a polymer network having a chain-length 


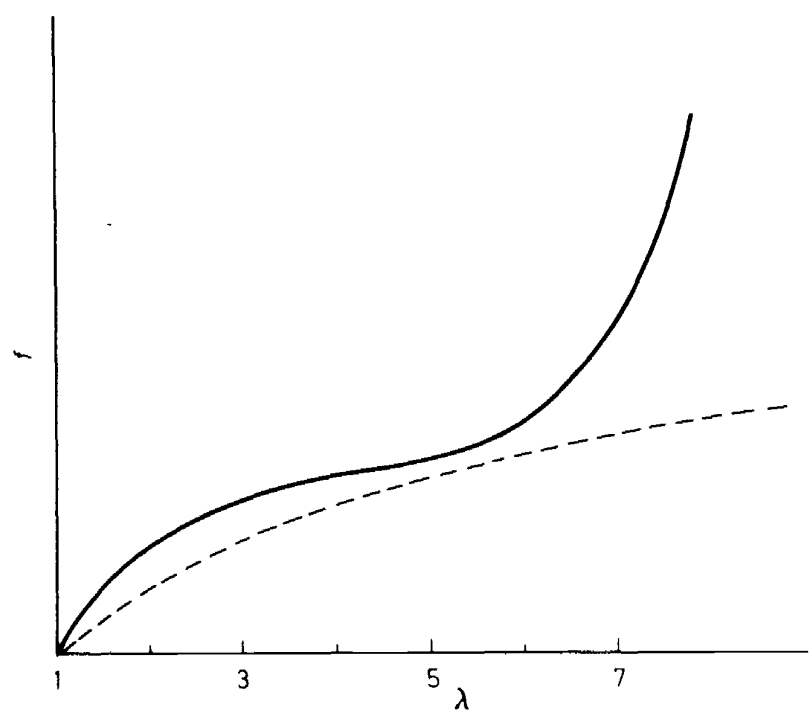

Figure 14. Schematic graph of the retractive force $f$ as function of the relative deformation $\lambda$. I ull curve: experimental results. Dotted curve: theory

distribution the segments of the shorter chains have a certain mean orientation, which is greater than that of the segments of the larger chains. while the free chain ends have no orientation at all in the equilibrium state. This is valid for independent chains, as is assumed in the statistical theory. But in reality the chains are not independent and we must assume a short-range order. The segments of the larger chains adjust their mean orientation to that of the shorter ones to some extent. so that the order can be maintained. This ordering state does not change with deformation, as Göritz and Müller have concluded from their measurements on crosslinked natural rubber ${ }^{35}$.

This additional orientation for the maintenance of the short-range order gives a decrease in entropy and therefore an addition to the retractive force. The birefringence will become greater as in the case of independent chains.

All experimental results regarding the $C_{2}$-term are in agreement with this model. The swelling by solvents of low molecular weight destroys the shortrange order in the polymer and decreases therefore the $C_{2}$-term. The decrease of the stress-optical coefficient of elastomers with swelling in isotropic solvents can also be explained in this way ${ }^{16,29,31,38,39}$. The decrease of the birefringence at constant strain with rising temperature is also caused by the loss of short-range order. The above mentioned polybutadiene network crosslinked by end-groups did not show a $C_{2}$-term because the chain-length distribution was very small and free chain ends were lacking ${ }^{22}$. The influence of the preparation of the networks and the type of deformation on the $C_{2}$-term can also be interpreted in this way. This qualitative explanation has recently been reinterpreted quantitatively ${ }^{38}$.

Two phenomena have therefore great influence on the elastic properties of crosslinked polymers: the chain-length distribution and the short-range order. 


\section{CONCLUSION}

Some remaining problems for research on rubber elasticity in the future may be mentioned briefly:

Experimentally we must try to construct better networks without entanglements, other physical crosslinks, heterogeneous regions and free chain ends. The chain-length distribution should be as narrow as possible. The question of volume constancy is very important. We should try to obtain exact results either by direct measurements or by calculation from constant-pressure measurements without rough approximations. How the crosslink density $v$ can be separated experimentally from the dilatation factor $\left\langle r^{2}\right\rangle /\left\langle r_{0}^{2}\right\rangle$ should be studied.

Theoretically the molecular interpretation of the modulus (cf. equation 16) must be reconsidered. We do not know if $v$ is the true crosslink density or only a measure of it. The front factor may vary between one and one-half Furthermore we must assume that rotational isomerism is an important but not the only molecular basis of the energy elastic contribution to the modulus. In the theoretical work the forces between the molecules must be considered too. A real network does not consist of isolated chains. In the theory of photoelasticity there remains the task of calculating the internal electric field and estimating its influence on birefringence. Despite many efforts, the consequences of a gaussian or non-gaussian network on the elasticity behaviour of a rubber are not fully understood.

\section{ACKNOWLEDGEMENTS}

The author thanks Drs E. E. Schäfer and J. Schwarz for careful measurements and valuable discussions. The provision of trans-polypentenamer by Bayer AG. Leverkusen. the preparation of natural rubber samples by Degussa, Kalscheuren, and the provision of endgroup-crosslinked polybutadiene by Dr G. Kraus, Phillips Petroleum, Bartlesville, is gratefully acknowledged.

\section{REFERENCES}

${ }^{1} \mathrm{~K}$. Dušek and W. Prins, Structure and Elasticity of Non-Crystalline Polymer Nerworks, Adv. Polymer Sci. Vol. 6, pp. 1-102 (1969).

2 T. A. J. Chompff and S. Newman, Polymer Networks, Proc. of the ACS Symposium on Highly Crosslinked Polymer Networks, Chicago 1970, Plenum: New York (1971).

${ }^{3}$ L. R. G. Treloar, The Physics of Rubber Elasticity, 2nd edn., Clarendon: Oxford (1958).

4 P. J. Flory, Principles of Polymer Chemistry, p. 434. Cornell University Press: Ithaca (1953).

5 L. R. G. Treloar in H. A. Stuart, Die Physik der Hochpolymeren, Vol. IV. 'The structure and mechanical properties of rubberlike materials', pp. 295-372. Springer: Berlin (1956).

6 A. J. Staverman and F. Schwarzl, Die Physik der Hochpolymeren, Vol. IV. 'Non-linear deformation behaviour of high polymers', pp. 126-164. Springer: Berlin (1956).

7 A. J. Staverman in S. Flügge. Encyclopedia of Physics, Vol. XIII. 'Thermodynamics of polymers', pp. 432 ff. Springer: Berlin (1962).

${ }^{8}$ L. R. G. Treloar, The Elasticity and Related Properties of Rubber, Rep. Progr. Phys. 36, 755-826 (1973).

9 P. J. Flory, A. Ciferri and C. A. J. Hoeve, J. Polymer Sci. 45, 235 (1960).

10 L. R. G. Treloar, Polymer, 10, 291 (1969); See also ref. 8, p. 792.

11 G. Allen, M. C. Kirkham, J. Padget and C. Price, Trans. Faraday Soc. 67, 1278 (1971); G. Allen, U. Bianchi and C. Price, Trans. Faraday Soc. 59, 2493 (1963).

12 E. E. Schäfer and G. Rehage, Makromol. Chem. 123, 91 (1969). 


\section{G. REHAGE}

13 J. A. Duiser and A. J. Staverman, On the Theory of Rubber Elasticity, in Physics of NonCrystalline Solids. ed. J. A. Prins, p. 376, North Holland: Amsterdam (1965).

14 W. Prins in Physics of Non-Crystalline Solids. ed. J. A. Prins, p. 360. North Holland : Amsterdam (1965).

15 W. Kuhn and H. Grün. Kolloid-Z. 101, 248 (1942).

16 K. Nagai, Conformational Statistics of Polymeric Chains, in Progress in Polymer Science Japan. Vol. I. p. 215. eds. M. Imoto and S. Onogi, Wiley: New York (1971).

17 cf. J. D. Ferry, Viscoelastic Properties of Polymers, 2nd edn. Wiley: New York (1970).

18 A. V. Tobolsky. D. W. Carlson. N. Indictor and M. S. Shen, J. Polymer Sci. 61, 523 (1962).

19 M. C. Shen and A. V. Tobolsky. J. Polymer Sci. A. 2.2513 (1964).

20 R. Caspary, Kautschuk und Gummi. Kunststoffe. 25. 249 (1972).

21 N. Buchholz, Diplomarbeit, Clausthal (1971).

22 G. Kraus and G. A. Moczygemba, J. Polymer Sci. A, 2. 277 (1964).

2.3 A. Opschoor and W. Prins, J. Polymer Sci. C, 16. 1095 (1967).

${ }^{24}$ M. V. Volkenstein. Configurational Statistics of Polymer Chains, Interscience: New York (1963).

25 T. M. Birshtein and O. B. Ptitsyn. Conformations of Macromolecules, Interscience: New York (1966).

26 P. J. Flory, Statistical Mechanics of Chain Molecules. Interscience: New York (1969).

27 cf. 23 and W. A. Wood. J. Res. Natl. Bur. Standards, 77A, 171 (1.973).

${ }^{28}$ V. Pollák. A. Romanov and K. Marciněin. IUPAC Symposium of Macromolecules. Abstract G 29, p. 444. Aberdeen (September 1973).

29 J. Schwarz, Ber. Bunsenges. Phys. Chem. 74, 848 (1970).

${ }^{30}$ G. Gebhard, Diplomarbeit. Clausthal (1971);

G. Gebhard, G. Rehage and J. Schwarz, publication in preparation.

${ }_{31}$ G. Rehage. E. E. Schäfer and J. Schwarz, Angew. Makromol. Chem. 16/17. 231 (1971).

32 J. Schwarz and G. Rehage. Kolloid-Z. u. Z. Polymere. 251. 689 (1973).

33 W. Borchard. M. Pyrlik and G. Rehage. Makromol. Chem. 145, 169 (1971).

${ }^{34}$ W. Borchard. G. Kalawrytinos, B. Mohadjer, M. Pyrlik and G. Rehage. Angew. Makromol. Chem. 29/30. 471 (1973).

35 D. Göritz and F. H. Müller. Kolloid-Z. u. Z. Polymere, 251, 679 (1973).

${ }^{36}$ M. Mooney, J. Appl. Phys., 11. 582 (1940).

${ }_{37}$ R. S. Rivlin. J. Appl. Phys., 18. 444 (1948).

38 J. Schwarz. Kolloid-Z. u. Z. Polvmere. 251, 215 (1973).

39 T. Ishikawa and K. Nagai. J. Polymer Sci. A-2, 7, 1123 (1969). 